International Journal of Bifurcation and Chaos, Vol. 27, No. 14 (2017) 1799001 (13 pages)

(C) World Scientific Publishing Company

DOI: $10.1142 / S 0218127417990012$

\title{
Author Index Volume 27 (2017)
}

Abdul-Hassan, K. M., see Tahir

Abdulla, M. U., see Abdulla

Abdulla, R. U., see Abdulla

Abdulla, U. G., Abdulla, R. U., Abdulla, M. U. \& Iqbal, N. H., Second minimal orbits, Sharkovski ordering and universality in chaos

Abdullah, M. A., see Tahir

Abouelmagd, E. I., Llibre, J. \& Guirao, J. L. G., Periodic orbits of the planar anisotropic Kepler problem

Acar, E., see Uguz

Adamatzky, A., see Dourvas

Adamatzky, A., Fredkin and Toffoli gates implemented in oregonator model of Belousov-Zhabotinsky medium

Adhyapok, P., Patra, M. \& Banerjee, S., The behavior of synchronized frequency in weakly coupled nonidentical periodic oscillators

Adusumilli, S. \& Van Gorder, R. A., Hyperchaos from a model of coupled stratospheretroposphere dynamics

Ahamed, A. I. \& Lakshmanan, M., Discontinuity induced Hopf and NeimarkSacker bifurcations in a memristive MuraliLakshmanan-Chua circuit

Ahmad, B., see Ren

Aihara, K., see Kamiyama

Aihara, K., see $X u$

Akgul, A., see Lai

Akgul, A., see Wang

Akgul, A., see Wang

Akin, H., see Uguz

Albuquerque, H. A., see Marcondes

Alombah, N. H., Fotsin, H. \& Romanic, K., Coexistence of multiple attractors, metastable chaos and bursting oscillations in a multiscroll memristive chaotic circuit

Alonso-Sanz, R., Losada, J. C. \& Porras, M. A., Bifurcation and chaos in the logistic map with memory

Alsaedi, A., see Odibat

Alsaedi, A., see Ren

Alshomrani, A. S., see Ghosh

Alvarez-Ramírez, M. \& García-Saldaña, J. D., On the homoclinic orbits of the Lü system
1750173

1730018

1730018

1730018

1750173

1750039

1750083

1750089

1750041

1750008

1730007

1730021

1750187

1730012

1730034

1750142

1750130

1750152

1750083

1750175

1750067

1750190

1750161

1750187

1750167

1750070
Amador, A., Freire, E., Ponce, E. \& Ros, J., On discontinuous piecewise linear models for memristor oscillators

Amandolese, X., see Virot

1730022

1750020

Ambrosio, B., Hopf bifurcation in an oscillatory-excitable reaction-diffusion model with spatial heterogeneity

An, F. X. \& Chen, F. Q., Multipulse orbits and chaotic dynamics of an aero-elastic FGP plate under parametric and primary excitations

Andres, J. \& Fišer, J., Sharkovsky-type theorems on $S^{1}$ applicable to differential equations

Arroyo, D., Hernandez, F. \& Orúe, A. B., Cryptanalysis of a classical chaos-based cryptosystem with some quantum cryptography features

Asheghi, R., see Moghimi

Aziz-Alaoui, M. A., see Odibat

Bagyalakshmi, M., Gangadharan, S. \& Ganesh, M., On chaotic behavior of temperature distribution in a heat exchanger

Bai, C., see Ren

Baik, J.-J., see Moon

Balanov, Z., see Hooton

Balasuriya, S., see Sulalitha Priyankara

Balibrea-Iniesta, F., see Lopesino

Banerjee, M., Ghorai, S. \& Mukherjee, N., Approximated spiral and target patterns in Bazykin's prey-predator model: Multiscale perturbation analysis

Banerjee, S., see Adhyapok

Banerjee, S., see Patra

Bao, B. \& Ma, J., Dynamic game behavior of retailers considering the quality of substitute products based on delay decision

1750065

1750050

1750042

1750004

1750055

1750161

1750168

1750076

1750176

1750087

1750156

1730001

1750038

1750008

1730033

1750206

1730051

1730002

Barton, D. A. W., see Renson

Bashkirtseva, I. \& Ryashko, L., Analysis of noise-induced chaos-order transitions in Rulkov model near crisis bifurcations

1730014

Bashkirtseva, I., Ryashko, L. \& Ryazanova, T., Analysis of noise-induced bifurcations in the stochastic tritrophic population system

Belghith, S., see Khraief Haddad
1750208 1750104 
Bella, G., A note on the Bogdanov-Takens bifurcation in the Romer model with learning by doing

Belozyorov, V. Ye., Reduction method for search of chaotic attractors in generic autonomous quadratic dynamical systems

Bertelle, C., see Lucas

Bi, Q., see Han

Biswas, S., see Ghosh

Biswas, S., see Saifuddin

Blé, G., see Valenzuela

Bollt, E., see Sulalitha Priyankara

Bouvry, P., see Changaival

Brown, T., see Ji

Buono, P.-L., van Veen, L. \& Frawley, E., Hidden symmetry in a Kuramoto-Sivashinsky initial-boundary value problem

Cai, N., see Ren

Cai, Q., see Gao

Campos-Cantón, E., see Gilardi-Velázquez

Cañete, J. F., see Luque

Cang, S. J., Wu, A., Wang, Z. \& Chen, Z., Distinguishing Lorenz and Chen systems based upon Hamiltonian energy theory

Cantin, G., Nonidentical coupled networks with a geographical model for human behaviors during catastrophic events

Cao, H. J., see Cheng

Cao, H. J., see Cheng

Cao, L., see Liu

Cao, Q. J., see $L i$

Cao, Z., see Chen

Carvalho, T. \& Llibre, J., On the periodic solutions of the five-dimensional Lorenz equation modeling coupled Rosby waves and gravity waves

Changaival, B., Rosalie, M., Danoy, G., Lavangnananda, K. \& Bouvry, P., Chaotic traversal (CHAT): Very large graphs traversal using chaotic dynamics

Chattopadhyay, J., see Ghosh

Chattopadhyay, J., see Saifuddin

Chemori, A., see Khraief Haddad

Chen, B., Chen, F. \& Martínez, G. J., Glider collisions in hybrid cellular automaton with memory rule $(43,74)$

Chen, E., Min, L. \& Chen, G., Discrete chaotic systems with one-line equilibria and their application to image encryption

Chen, F. Q., see An

Chen, F., see Chen

Chen, F., see $X u$

Chen, G., Kuznetsov, N. V., Leonov, G. A. \& Mokaev, T. N., Hidden attractors on one path: Glukhovsky-Dolzhansky, Lorenz, and Rabinovich systems

Chen, G., see Chen

Chen, G., see Min

Chen, G., see Nazarimehr
Chen, G., see $W u$

1750127

Chen, G., see Zhang

1750002

Chen, H., Cao, Z., Li, D. \& Xie, J., Global 1750155 analysis on a discontinuous dynamical system

1750036

1750219

1750051

1750167

1730027

1750034

1750156

1750215

1750066

1750136

1750187

1750059

1730031

1730051

1750024

1750213

1750178

1730009

1750033

1750009

1750078

1750090

Chen, L., see Liu

Chen, S., see Guo

Chen, X., see Sun

Chen, Y.-A., see Zhang

Chen, Z., see Cang

Chen, Z., see Sun

Chen, Z., see Zhou

Cheng, L. F. \& Cao, H. J., Effect of higher order terms on the bifurcation structure of coupled Rulkov neurons

Cheng, L. \& Cao, H. J., Synchronization dynamics of two heterogeneous chaotic Rulkov neurons with electrical synapses

Cheng, X. \& She, Z., Study on chaotic behavior of the restricted four-body problem with an equilateral triangle configuration

Chialva, U. \& Reartes, W., Heteroclinic cycles in a competitive network

1750078

1730026

1750124

1750073

1750075

1750024

1750197

1750021

1750178

1730009

1750026

1730044

Cho, K. \& Miyano, T., Design and test of pseudorandom number generator using a star network of Lorenz oscillators

1750215

1750167

1730027

1750104

1750082

1750046

1750050

1750082

1750217

1750115

1750046

1750029

1750221
Choi, H., see Mannan

Chu, Z., see Wang

Chua, L. O., see Stankevich

Chua, L., see Itoh

Chua, L., see Itoh

Chua, L., see Mannan

Chua, L., see Rajamani

Church, K. E. M. \& Liu, X., Bifurcation analysis and application for impulsive systems with delayed impulses

Churilov, A. N., Medvedev, A. \& Zhusubaliyev, Z. T., Discrete-time mapping for an impulsive Goodwin oscillator with three delays

Cicek, S., see Wang

Ciurdariu, L., see Tigan

Comassetto, G. F., see Marcondes

Contassot-Vivier, S., Couchot, J.-F., Guyeux, C. \& Heam, P.-C., Random walk in a Ncube without Hamiltonian cycle to chaotic pseudorandom number generation: Theoretical and practical considerations

Córdoba, A., see Luque

Corson, N., see Odibat

Cotsaftis, M., see Lucas

Couchot, J.-F., see Contassot-Vivier

Dai, B., see Han

Danca, M.-F. \& Fečkan, M., On numerical integration of discontinuous dynamical systems

1750184

1730011

1750139

1730038

1730035

1730005

1730011

1730015

1750186

Dang, W.-D., see Gao

Dang, W.-D., see Gao

Danoy, G., see Changaival
1750014

1730051

1750161

1750219

1750014

1750088

1750218

1750059

1750123

1750215 
Dar, M. R., Kant, N. A. \& Khanday, F. A., Realization of integrable incommensuratefractional-order-Rössler-system design using operational transconductance amplifiers (OTAs) and its experimental verification

1750077

1730003

Degens, H., see Ramos

Delgado, J., Hernández-Martínez, L. I. \& Pérez-López, J., Global bifurcation map of the homogeneous states in the Gray-Scott model

Demian, A. S. \& Wiggins, S., Detection of periodic orbits in Hamiltonian systems using Lagrangian descriptors

Deng, W., Liao, X. \& Dong, T., Dynamical behaviors in complex-valued Love model with or without time delays

de Oliveira, P. P. B., see Ruivo

De Santis, E., Sadeghian, A. \& Rizzi, A., A smoothing technique for the multifractal analysis of a medium voltage feeders electric current

Di Bernardo, M., see Erazo

Ding, H., see Zhang

Ding, H., see Zhang

Ding, H., see Zhang

Ding, H., see Zhang

Ding, Q., see Zhang

Ding, X., see $W u$

Do, Y., see Gao

Domański, P. D., Multifractal properties of process control variables

Dong, T. \& Xia, L., Stability and Hopf bifurcation of a reaction-diffusion neutral neuron system with time delay

Dong, T., see Deng

Dou, G., see Guo

Dou, G., see Zhang

Dourvas, N. I., Sirakoulis, G. Ch. \& Adamatzky, A., Cellular automaton Belousov-Zhabotinsky model for binary full adder

Du, Y., Guo, Y. \& Xiao, P., Freely-moving delay induces periodic oscillations in a structured SEIR model

Du, Z., see $L i$

Duan, J. Y., Jiang, G. P. \& Yang, H., A novel multiple-access correlation-delayshift-keying

Duan, L., see Lü

Duan, L., see Wang

Dubey, B., see Maiti

Dudkowski, D., Prasad, A. \& Kapitaniak, T., Perpetual points: New tool for localization of coexisting attractors in dynamical systems

Elhadj, Z., see Fareh

El-Nashar, H. F., Conditions and linear stability analysis at the transition to synchronization of three coupled phase oscillators in a ring

1750200

1730028

1730041

1750069

1750086

1750096

1750140

1750212

1750165

1750123

1750094

1750214

1750200

1730047

1750148

1750122

1750162

1750025

1750172

1750181

1750174

1750079

1750095
Erazo, C., Homer, M. E., Piiroinen, P. T. \& Di Bernardo, M., Dynamic cell mapping algorithm for computing basins of attraction in

1750089 Gao, Z.-K., Li, S., Dang, W.-D., Yang, Y.-X.,

1750063 planar Filippov systems

Euzzor, S., see Ginoux

Fadeev, S. I., see Kogai

Fahmy, H. A. H., see Sayed

Falconi, M., see Valenzuela

Falcão, M. I., Miranda, F., Severino, R. \& Soares, M. J., Iteration of quadratic maps on coquaternions

Faranda, D., see Virot

Fareh, H. \& Elhadj, Z., Necessary and sufficient conditions for the occurrence of bisecting bifurcations in the general $2 D$ piecewiselinear mapping

Farghaly, A. A. M., see Mahmoud

Fečkan, M., see Danca

Feng, Z., see Jiang

Feng, Z., see Yang

Ferrer, J., Peña, M. \& Susin, A., Bifurcation diagram of saddle/spiral bimodal linear systems

Fišer, J., see Andres

Fotsin, H., see Alombah

Fournier-Prunaret, D., see Leonel Rocha

Frawley, E., see Buono

Freire, E., see Amador

Fu, X., see Yan

Gaitonde, A., see Huntley

Galias, Z., Numerical study of multiple attractors in the parallel inductor-capacitormemristor circuit

Ganesh, M., see Bagyalakshmi

Gangadharan, S., see Bagyalakshmi

Gao, Y., Peng, L. \& Liu, C., Bifurcation of limit cycles from a class of piecewise smooth systems with two vertical straight lines of singularity

Gao, Y., see Wang

Gao, Z., see Guo Do, Y. \& Grebogi, C., Wavelet multiresolution complex network for analyzing multivariate nonlinear time series

1730041

1750220

1730006

1730004

1750034

1730039

1750020

1750079

1750144

1750218

1750222

1750015

1750005

1750042

1750067

1730030

1750136

1730022

1750032

1750061

1730036

1750168

1750168

1750157

1750119

1730047

1750123

Gao, Z.-K., Zhang, S.-S., Dang, W.-D., Li, S. \& Cai, Q., Multilayer network from multivariate time series for characterizing nonlinear flow behavior

Garay, B., Siegmund, S., Trostorff, S. \& Waurick, M., Some remarks on local activity and local passivity

García, I. A. \& Llibre, J., Classical planar algebraic curves realizable by quadratic polynomial differential systems

García-Garrido, V. J., see Lopesino

García-Saldaña, J. D., see Alvarez-Ramírez

Ghorai, S., see Banerjee

Ghosh, K., Biswas, S., Samanta, S., Tiwari, P. K., Alshomrani, A. S. \& Chattopadhyay,

1750059 
J., Effect of multiple delays in an ecoepidemiological model with strong Allee effect

Gilardi-Velázquez, H. E., Ontañón-García, L. J., Hurtado-Rodriguez, D. G. \& CamposCantón, E., Multistability in piecewise linear systems versus eigenspectra variation and round function

Giné, J., Liénard equation and its generalizations

Ginoux, J.-M., Meucci, R. \& Euzzor, S., Torus breakdown and homoclinic chaos in a glow discharge tube

Glimm, T., Zhang, J. \& Shen, Y.-Q., Stability of Turing-type patterns in a reactiondiffusion system with an external gradient

Gohara, K., see Hirakawa

Golpayegani, S. M. R. H., see Nazarimehr

Golpayegani, S. M. R. H., see Nazarimehr

Goncu, E. \& Yalcin, M. E., Cellular automata with random memory and its implementations

Grebogi, C., see Gao

Grebogi, C., see Ren

Gritli, H., see Khraief Haddad

$\mathrm{Gu}, \mathrm{A} . \& \mathrm{Li}, \mathrm{Y} .$, A combined criterion for existence and continuity of random attractors for stochastic lattice dynamical systems

$\mathrm{Gu}$, E.-G., Bifurcations and chaos for $2 D$ discontinuous dynamical model of financial markets

Gu, H. G., see Jia

Guirao, J. L. G., see Abouelmagd

Guo, M., Xue, Y., Gao, Z., Zhang, Y., Dou, G. \& Li, Y., Dynamic analysis of a physical SBT memristor-based chaotic circuit

Guo, M., see Zhang

Guo, S., see Sun

Guo, S., see Yan

Guo, S., see Zou

Guo, X. Y., Zhang, Y., Zhang, W., Sun, L. \& Chen, S., Nonlinear dynamics of Z-shaped folding wings with 1:1 inner resonance

Guo, Y., see Du

Gupta, M. K. \& Yadav, C. K., Jacobi stability analysis of Rössler system

Guyeux, C., see Contassot-Vivier

Haase, M., see Rabbow

Hajipour, A. \& Tavakoli, H., Dynamic analysis and adaptive sliding mode controller for a chaotic fractional incommensurate order financial system

Hamdi, B. \& Hassen, S., A new hypersensitive hyperchaotic system with no equilibria

Han, B., see Zhang

Han, B.-S., see Moon

Han, H., see $X u$

Han, M., see Xiong

Han, R. \& Dai, B., Cross-diffusion induced turing instability and amplitude equation for a toxic-phytoplankton-zooplankton model with nonmonotonic functional response

1750088

1750167

Han, X. J., Zhang, C., Yu, Y. \& Bi, Q., Boundary-crisis-induced complex bursting patterns in a forced cubic map

Hantáková, J., Iteration problem for distributional chaos

1730031 Harkin, J., see Liu

Hassen, S., see Hamdi

1750081 He, J., Yu, S. \& Lü, J., Constructing higher-dimensional nondegenerate hyperchaotic systems with multiple controllers

1750220

He, J., see Qiu

$\mathrm{He}, \mathrm{X}$., see $W u$

He, Y., see Liu

1750003

1730040

1750201

1750023

1730017

1750123

1750076

1750104

1750019

Heam, P.-C., see Contassot-Vivier

Hémon, P., see Virot

Hernandez, F., see Arroyo

Hernández-Martínez, L. I., see Delgado

Hirakawa, T., Suzuki, H., Gohara, K. \& Yamamoto, Y., Inverse proportional relationship between switching-time length and fractal-like structure for continuous tracking movement

Hoang, D. V., see Wang

Hoang, T. M., see Tahir

Hoff, A., see Marcondes

Homer, M. E., see Erazo

Hooton, E., Balanov, Z., Krawcewicz, W. \& Rachinskii, D., Noninvasive stabilization of periodic orbits in $\mathrm{O}_{4}$-symmetrically coupled systems near a Hopf bifurcation point

1750185

1750113

1750039

Hoover, W. G., see Sprott

Hosseini, S. S., Nazarimehr, F. \& Jafari, S., Investigation of seasonal and latitudinal effects on the expression of clock genes in drosophila

1730047

1750148

1750073

1750105

1750049

1750124

1750122

1750056

1750014

1750001

Hou, X., see $L i$

$\mathrm{Hu}$, J., see $Q i$

$\mathrm{Hu}, \mathrm{W}$. , see $L i$

$\mathrm{Hu}, \mathrm{W}$. , see Yuan

$\mathrm{Hu}$, W., see Zhang

Huan, S.-M., Existence and stability of invariant cones in 3-dim homogeneous piecewise linear systems with two zones

Huan, S.-M., Existence of invariant cones in general 3-dim homogeneous piecewise linear differential systems with two zones

Huang, B., Bifurcation of limit cycles from the center of a quintic system via the averaging method

1750198 Huang, T., see $W u$

Huang, X., see Wang

Huang, Z.-Z., see Ren

1750064

1750212

1750176

1730046

1750126

Huntley, S., Jones, D. \& Gaitonde, A., Bifurcation tracking for high Reynolds number flow around an airfoil

Hurtado-Rodriguez, D. G., see GilardiVelázquez

Hutchinson, J. W., see Thompson
1750051

1750183

1750033

1750064

1750146

1750040

1750031

1750171

1750014

1750020

1750004

1730024

1730040

1750130

1750173

1750175

1730041

1750087

1730037

1750153

1750018

1750216

1750160

1750210

1750140

1750007

1750189

1750072

1750031

1750209

1750076

1750061

1730031

1730048 
Iqbal, N. H., see Abdulla

Iqbal, N., see Liu

Itoh, M. \& Chua, L., Chaotic oscillation via edge of chaos criteria

Itoh, M. \& Chua, L., Dynamics of Hamiltonian systems and memristor circuits

Jafari, S., see Hosseini

Jafari, S., see Nazarimehr

Jafari, S., see Nazarimehr

Jafari, S., see Nazarimehr

Jafari, S., see Nazarimehr

Jafari, S., see Pham

Jafari, S., see Pham

Jafari, S., see Pham

Jafari, S., see Wang

Janková, K., see Kováč

Jeong, B., see Kim

Ji, J. C. \& Brown, T., Periodic and chaotic motion of a time-delayed nonlinear system under two coexisting families of additive resonances

Jia, B. \& Gu, H. G., Dynamics and physiological roles of stochastic firing patterns near bifurcation points

Jia, Q., On techniques towards chaos preservation and construction

Jia, R., see Yang

Jiang, G. P., see Duan

Jiang, J. \& Yu, P., Multistable phenomena involving equilibria and periodic motions in predator-prey systems

Jiang, N., see Zhang

Jiang, T., Yang, Z. \& Jing, Z., Bifurcations and chaos in the Duffing equation with parametric excitation and single external forcing

Jiang, W., see Yuan

Jiang, X., She, Z., Feng, Z. \& Zheng, X., Bifurcation analysis of a predator-prey system with ratio-dependent functional response

Jiang, X., see Wang

Jiang, Z. \& Wang, L., Global Hopf bifurcation for a predator-prey system with three delays

Jimenez Beristain, L., see Starkov

Jin, W., see $X u$

Jin, Y., Yang, S., Liu, Y., Xie, D. \& Zhang, N., Bifurcations of heteroclinic loop with twisted conditions

Jing, Z., see Jiang

Jones, D., see Huntley

Jones, D., see Ramos

Kamiyama, K., Komuro, M. \& Aihara, K., Homoclinic cycle bifurcations in planar maps

Kang, X., see Yan

Kant, N. A., see Dar

Kapitaniak, T., see Dudkowski

Kapitaniak, T., see Nazarimehr

Kapitaniak, T., see Pham

Kapitaniak, T., see Pham
1730018

1730026

1730035

1730005

1750153

1750201

1750221

1750023

1750037

1750053

1750097

1750138

1750152

1750117

1750006

1750066

1750113

1750191

1750199

1750025

1750043

1750169

1750125

1750028

1750222

1750010

1750108

1750151

1750217

1750120

1750125

1750061

1730003

1730012

1750147

1750077

1750063

1750221

1750053

1750097
Kapitaniak, T., see Pham

1750138

1730034

1750201

Kauffman, L. H., see Nazarimehr

1750055

Kengne, J., Nguomkam Negou, A. \& Njitacke, Z. T., Antimonotonicity, chaos and multiple attractors in a novel autonomous jerk circuit

Khanday, F. A., see Dar

Khlebodarova, T. M., see Kogai

Khraief Haddad, N., Belghith, S., Gritli, H. \& Chemori, A., From Hopf bifurcation to limit cycles control in underactuated mechanical systems

Kim, H., Seo, J., Jeong, B. \& Min, C., An experiment of the Malkus-Lorenz waterwheel and its measurement by image processing

Kim, H., see Mannan

Kim, H., see Rajamani

Kingni, S. T., see Pham

Kogai, V. V., Likhoshvai, V. A., Fadeev, S. I. \& Khlebodarova, T. M., Multiple scenarios of transition to chaos in the alternative splicing model

Kolokolov, Yu. \& Monovskaya, A., A practiceoriented bifurcation analysis for pulse energy converters: A stability margin

Komuro, M., see Kamiyama

Kong, C. \& Liu, X.-B., Noise-induced chaos in a piecewise linear system

Kou, K. I., see $L i$

Kou, K. \& Li, J., Exact traveling wave solutions of the Krichever-Novikov equation: A dynamical system approach

Kou, L., see $L i$

Kou, L., see $L i$

Kousaka, T., see Matsushita

Kovalev, D. P. \& Kovalev, P. D., Synchronization of long ocean waves by coastal relief on the southeast shelf of Sakhalin island

Kovalev, P. D., see Kovalev

Kováč, J. \& Janková, K., Random dynamical systems generated by two Allee maps

Krawcewicz, W., see Hooton

Kumari, N., see Sharma

Kurokawa, H., see Matsushita

Kuznetsov, N. V., see Chen

Kuznetsov, N. V., see Nazarimehr

Kuznetsov, N. V., see Stankevich

Lai, Q., Akgul, A., Zhao, X.-W. \& Pei, H., Various types of coexisting attractors in a new $4 D$ autonomous chaotic system

Lakshmanan, M., see Ahamed

Lavangnananda, K., see Changaival

Lăzureanu, C., The real-valued Maxwell-Bloch equations with controls: From a HamiltonPoisson system to a chaotic one

1750100

1750077

1730006

1750104

1750006

1730011

1730015

1750053

1730006

1750134

1730012

1750137

1750044

1750058

1750224

1750131

1750101

1750195

1750195

1750117

1750087

1750170

1750101

1750115

1750221

1730038

1750142

1730021

1750215

Léger, A., see Li

Lei, F., see Wang
1750143

1750009

1750139 
Lei, Y., Zheng, F. \& Shao, X., Chaos and chaos control of the Frenkel-Kontorova model with dichotomous noise

Leo Kingston, S. \& Thamilmaran, K., Bursting oscillations and mixed-mode oscillations in driven Liénard system

Leonel Rocha, J., Taha, A.-K. \& FournierPrunaret, D., Homoclinic and big bang bifurcations of an embedding of $1 D$ Allee's functions into a $2 D$ diffeomorphism

Leonel Rocha, J., see Silva

Leonov, G. A., see Chen

Leonov, G. A., see Nazarimehr

Leonov, G. A., see Stankevich

Leonov, G., Hausdorff-Lebesgue dimension of attractors

Leta, T. D. \& Li, J., Various exact soliton solutions and bifurcations of a generalized Dullin-Gottwald-Holm equation with a power law nonlinearity

Li, B., see Sui

Li, C. \& Sprott, J. C., How to bridge attractors and repellors

Li, C., Liu, H., Zhang, T. \& Yan, F., Hopf bifurcation analysis of a gene regulatory network mediated by small noncoding RNA with time delays and diffusion

Li, C., Sprott, J. C., Hu, W. \& Xu, Y., Infinite multistability in a self-reproducing chaotic system

Li, C., see Nazarimehr

$\mathrm{Li}, \mathrm{C}$., see $W u$

Li, C., see $X u$

Li, D., see Chen

Li, H., see Yuan

Li, J. \& Kou, K. I., Dynamics of traveling wave solutions to a new highly nonlinear shallow water wave equation

Li, J. \& Zhang, T., Bifurcations and exact solutions of the equation of barotropic FRW cosmologies

Li, J., Kou, L. \& Wang, D., Unique normal form for a class of three-dimensional nilpotent vector fields

Li, J., Kou, L., Wang, D. \& Zhang, W., Unique normal form and the associated coefficients for a class of three-dimensional nilpotent vector fields

Li, J., see Kou

Li, J., see Leta

Li, J., see Zhang

Li, J., see Zhou

Li, J., Exact solutions in the invariant manifolds of the generalized integrable HénonHeiles system and exact traveling wave solutions of Klein-Gordon-Schrödinger equations

Li, N., see Wang

Li, N., see Zhang

Li, S., see Gao
Li, S., see Gao

1750123

Li, S., see Luo

1750203

1750052

Li, T., see $M a$

Li, W., see Zhang

Li, W., see Zhang

1730025

Li, W., see Zhou

Li, W.-T., see $M a$

$\mathrm{Li}$, X., see Wang

Li, X., see Zhang

1730030

1730020

1750115

1750221

1730038

1750164

1750129

1750196

1750149

1750194

1750160

1750221

1750031

1750062

1750078

1750210

1750044

Li, Y. C. \& Yang, H., A mathematical model of demand-supply dynamics with collectability and saturation factors

Li, Y., Wang, J. \& Hou, X., Stripe and spot patterns for general Gierer-Meinhardt model with common sources

Li, Y., Yuan, L. \& Du, Z., Bifurcation of nonhyperbolic limit cycles in piecewise smooth planar systems with finitely many zones

Li, Y., see Guo

Li, Y., see Gu

Li, Y., see Long

Li, Y., see Sun

Li, Y., see Wang

Li, Y., see $X u$

Li, Y., see Zhang

Li, Z. X., Cao, Q. J. \& Léger, A., Threshold of multiple stick-slip chaos for an archetypal self-excited SD oscillator driven by moving belt friction

Liang, F. \& Wang, D., Limit cycle bifurcations near a piecewise smooth generalized homoclinic loop with a saddle-fold point

Liang, X., see $Q i$

Liao, B., see Lin

Liao, M., Su, Y. \& Zhou, Y., Oscillation reconstruction and bifurcation analysis of a Drillbit-Rock vibro-impact system

Liao, X., see Deng

Liao, X., see Sun

Liao, X., see Zhang

$1750080 \quad$ Liao, X., see Zhang

Likhoshvai, V. A., see Kogai

Lima, M. F. S., Pessoa, C. \& Pereira, W. F.,

$1750131 \quad$ Limit cycles bifurcating from a period annulus in continuous piecewise linear differential systems with three zones

Lin, J., see Liu

1750224

1750058

1750129

1750205

1750188

1750012

1750139

1750169

1750059

1750098

1750096

1750140

1750193

1750011

1750110

1750169

1750016

1750018

1750162

1730047

1750019

1750054

1750099

1750209

1730046

1750148

1750009

1750071

1750180

1750207

1750013

1750200

1750073

1750074

1750075

1730006

1750022

1750103

Lin, P., Wang, C. \& Wang, M., Bifurcation predication in axial compressors with nonuniform inflow via deterministic learning

Lin, X., Liao, B. \& Zeng, C., The onset of chaos via asymptotically perioddoubling cascade in fractional order Lorenz system

Lin, Z., Yu, S. \& Lü, J., A novel approach for constructing one-way hash function based on a message block controlled 8D hyperchaotic map

Lin, Z., see Qiu
1750159

1750207

1750106

1750040 
Liu, B., Wu, R., Iqbal, N. \& Chen, L., Turing patterns in the Lengyel-Epstein system with superdiffusion

Liu, B., see Liu

Liu, C., see Gao

Liu, C., see Yang

Liu, H., see $L i$

Liu, H., see Wang

Liu, J., see Liu

Liu, L. F., Lin, J., Miao, S. \& Liu, B., A double perturbation method for reducing dynamical degradation of the digital Baker map

Liu, S., see Wang

Liu, W., Sun, K., He, Y. \& Yu, M., Color image encryption using three-dimensional sine ICMIC modulation map and DNA sequence operations

Liu, W., see Shen

Liu, X. \& Zhang, T., Bogdanov-Takens and triple zero bifurcations of coupled van der Pol-Duffing oscillators with multiple delays

Liu, X., see Church

Liu, X.-B., see Kong

Liu, Y., Luo, Y., Song, S., Cao, L., Liu, J. \& Harkin, J., Counteracting dynamical degradation of digital chaotic Chebyshev map via perturbation

Liu, Y., see Jin

Liu, Y., see Wang

Liu, Y., see Zhang

Llibre, J., see Abouelmagd

Llibre, J., see Carvalho

Llibre, J., see García

Llibre, J., see Tigan

Long, M., Li, Y. \& Peng, F., Integrity verification for multiple data copies in cloud storage based on spatiotemporal chaos

Lopesino, C., Balibrea-Iniesta, F., GarcíaGarrido, V. J., Wiggins, S. \& Mancho, A. M., A theoretical framework for Lagrangian descriptors

López, Á. G., Seoane, J. M. \& Sanjuán, M. A. F., Bifurcation analysis and nonlinear decay of a tumor in the presence of an immune response

Losada, J. C., see Alonso-Sanz

Lou, W., see $M a$

Lozoya-Ponce, R. E., see Ontañón-García

Lu, B., see Wang

Lü, J., see $H e$

Lü, J., see Lin

Lü, J., see Qiu

Lü, J., see $X u$

Lü, Z. S., Zhao, C., Zhang, B. \& Duan, L., Multitime scale study of bursting activities in the pre-Bötzinger complex

Lu, T., see $W u$

Lucas, I., Cotsaftis, M. \& Bertelle, C., Heterogeneity and self-organization of complex

systems through an application to financial market with multiagent systems

1730026

1750103

1750157

1750199

1750194

1750119

1750033

1750103

1750010

1750171

1750204

1750133

1750186

1750137

1750033

1750120

1750048

1750155

1750039

1750090

1750141

1750068

1750054

1730001

1750223

1750190

1750158

1750093

1750010

1750146

1750106

1750040

1750062

1750172

1750165
Luo, J. \& Zhao, Y., Stability and bifurcation analysis in a predator-prey system with constant harvesting and prey group defense

Luo, P., see Zhang

Luo, P., see Zhang

Luo, S., Li, S. \& Tajaddodianfar, F., Chaos and adaptive control of the fractional-order magnetic-field electromechanical transducer

Luo, Y., see Liu

Luque, A., Barbancho, J., Cañete, J. F. \& Córdoba, A., Phase shadows: An enhanced representation of nonlinear dynamic systems

Lv, Y., Xiong, T. \& Yi, W., Multistability in a simplified underwater supercavity system

Lynch, S., see Ramos

Lyu, J., see Xiong

Ma, J. H., Yang, W. \& Lou, W., Research on bifurcation and chaos in a dynamic mixed game system with oligopolies under carbon emission constraint

Ma, J., Li, T. \& Ren, W., Research on the complexity of dual-channel supply chain model in competitive retailing service market

Ma, J., see Bao

Ma, J., see Ren

Ma, J., see Wang

Ma, Z. D. \& Ning, L. J., Bifurcation regulations governed by delay self-control feedback in a stochastic birhythmic system

Ma, Z.-P., Li, W.-T. \& Wang, Y.-X., Spatiotemporal patterns induced by crossdiffusion in a three-species food chain model

Maciel, A. L., see Messias

Mahmoud, G. M., Farghaly, A. A. M. \& Shoreh, A. A.-H., A technique for studying a class of fractional-order nonlinear dynamical systems

Maiti, A. P. \& Dubey, B., Stability and bifurcation of a Fishery model with CrowleyMartin functional response

Manchein, C., see Marcondes

Mancho, A. M., see Lopesino

Mannan, Z. I., Choi, H., Rajamani, V., Kim, H. \& Chua, L., Chua corsage memristor: Phase portraits, basin of attraction, and coexisting pinched hysteresis loops

Mannan, Z. I., see Rajamani

Marcondes, D. W. C., Comassetto, G. F., Pedro, B. G., Vieira, J. C. C., Hoff, A., Prebianca, F., Manchein, C. \& Albuquerque, H. A., Extensive numerical study and circuitry implementation of the Watt Governor model

Martínez, G. J., see Chen

Matsushita, H., Tomimura, Y., Kurokawa, H. \& Kousaka, T., Period doubling bifurcation
1750219

1750179

1750069

1750140

1750203

1750033

1730051

1750121

1730003

1750128

1750158

1750098

1750206

1750187

1750030

1750202

1750011

1750102

1750144

1750174

1750175

1730001

1730011

1730015

1750175 1750082 
point detection strategy with nested layer particle swarm optimization

Medvedev, A., see Churilov

Messias, M. \& Maciel, A. L., On the existence of limit cycles and relaxation oscillations in a $3 D$ van der Pol-like memristor oscillator

Meucci, R., see Ginoux

Miao, J., see Wang

Miao, S., see Liu

Miao, Y., see Yuan

Min, C., see Kim

Min, L. \& Chen, G., Generalized stability in an array of nonlinear dynamic systems with applications to chaotic CNN

Min, L., see Chen

Mindlin, G. B., see Roulet

Miranda, F., see Falcão

Mireles James, J. D. \& Murray, M., Chebyshev-Taylor parameterization of stable/unstable manifolds for periodic orbits: Implementation and applications

Miyano, T., see Cho

Mliki, E., see Wang

Moghimi, P., Asheghi, R. \& Kazemi, R., On the number of limit cycles bifurcated from some Hamiltonian systems with a double homoclinic loop and a heteroclinic loop

Mokaev, T. N., see Chen

Mondal, A., see Upadhyay

Monovskaya, A., see Kolokolov

Moon, S., Han, B.-S., Park, J., Seo, J. M. \& Baik, J.-J., Periodicity and chaos of highorder Lorenz systems

Mora, A., see Rabbow

Moroz, I., see Wei

$\mathrm{Mu}, \mathrm{C}$., see Zhang

$\mathrm{Mu}, \mathrm{C}$., see Zhang

$\mathrm{Mu}, \mathrm{P}$. , see Zhang

Mukherjee, N., see Banerjee

Murray, M., see Mireles James

Musafirov, E. V., Perturbations of the Lanford system which do not change the reflecting function

Nazarimehr, F., Jafari, S., Chen, G., Kapitaniak, T., Kuznetsov, N. V., Leonov, G. A., Li, C. \& Wei, Z., A tribute to J. C. Sprott

Nazarimehr, F., Jafari, S., Golpayegani, S. M. R. H. \& Kauffman, L. H., Investigation of bifurcations in the process equation

Nazarimehr, F., Jafari, S., Golpayegani, S. M. R. H. \& Sprott, J. C., Categorizing chaotic flows from the viewpoint of fixed points and perpetual points

Nazarimehr, F., Saedi, B., Jafari, S. \& Sprott, J. C., Are perpetual points sufficient for locating hidden attractors?

Nazarimehr, F., see Hosseini

Neild, S. A., see Renson

Nguomkam Negou, A., see Kengne

Ning, L. J., see $M a$
1750101

1750182

1750102

1750220

1750181

1750103

1750210

1750006

1750029

1750046

1730045

1730039

1730050

1750184

1750152

1750055

1750115

1730019

1750134

1750176

1750001

1730008

1750074

1750075

1750169

1750038

1730050

1750154

1750221

1750201

1750023

1750037

1750153

1730002

1750100

1750202
Njitacke, Z. T., see Kengne

1750100

Odibat, Z., Corson, N., Aziz-Alaoui, M. A. \& Alsaedi, A., Chaos in fractional order cubic Chua system and synchronization

1750161

Ontañón-García, L. J. \& Lozoya-Ponce, R. E., Analog electronic implementation of unstable dissipative systems of Type I with multiscrolls displaced along space

Ontañón-García, L. J., see Gilardi-Velázquez

Orúe, A. B., see Arroyo

Osipov, A. V. \& Söderbacka, G., Poincaré map construction for some classic two predatorsone prey systems

Pan, W., see Zhang

Panda, L. N., see Sahoo

Park, J., see Moon

Patra, M. \& Banerjee, S., Bifurcation of quasiperiodic orbit in a $3 D$ piecewise linear map

Patra, M., see Adhyapok

Pedro, B. G., see Marcondes

Pei, H., see Lai

Peña, M., see Ferrer

Pender, J., Rand, R. H. \& Wesson, E., Queues with choice via delay differential equations

Peng, F., see Long

Peng, L., see Gao

Pereira, W. F., see Lima

Pérez-López, J., see Delgado

Pesek, L., see Pust

Pessoa, C., see Lima

Pham, V.-T., Jafari, S., Kapitaniak, T., Volos, C. \& Kingni, S. T., Generating a chaotic system with one stable equilibrium

Pham, V.-T., Jafari, S., Volos, C. \& Kapitaniak, T., Different families of hidden attractors in a new chaotic system with variable equilibrium

Pham, V.-T., Wang, X., Jafari, S., Volos, C. \& Kapitaniak, T., From Wang-Chen system with only one stable equilibrium to a new chaotic system without equilibrium

Pham, V.-T., see Tahir

Pham, V.-T., see Wang

Pham, V.-T., see Wang

Piiroinen, P. T., see Erazo

Plath, P. J., see Rabbow

Pohit, G., see Sahoo

Ponce, E., see Amador

Porras, M. A., see Alonso-Sanz

Prasad, A., see Dudkowski

Prebianca, F., see Marcondes

Pust, L. \& Pesek, L., Blades forced vibration under aero-elastic excitation modeled by van der Pol

Qi, G. \& Hu, J., Force analysis and energy operation of chaotic system of permanentmagnet synchronous motor

Qi, G. \& Liang, X., Mechanism and energy cycling of the Qi four-wing chaotic system
1750093

1730031

1750004

1750116

1750169

1750017

1750176

1750008

1750175

1750142

1750005

1730016

1750054

1750157

1750022

1730024

1750166

1750022

1750053

1750138

1750097

1750173

1750130

1750152

1730041

1750001

1750017

1730022

1750190

1750063

1750175

1750166

1750216

1750180
1730033 
Qiao, Y., see Wang

Qin, B., see Sun

Qiu, M., Yu, S., Wen, Y., Lü, J., He, J. \& Lin, Z., Design and FPGA implementation of a universal chaotic signal generator based on the verilog HDL fixed-point algorithm and state machine control

Quyen, N. X., On the study of a quadrature DCSK modulation scheme for cognitive radio

Rabbow, T. J., Plath, P. J., Mora, A. \& Haase, M., Selforganized structure formation in organized microstructuring by laserjet etching

Rachinskii, D., see Hooton

Radwan, A. G., see Sayed

Rajamani, V., Sah, M. PD., Mannan, Z. I., Kim, H. \& Chua, L., Third-order memristive Morris-Lecar model of barnacle muscle fiber

Rajamani, V., see Mannan

Rajapakse, I. \& Smale, S., The pitchfork bifurcation

Ramos, J., Lynch, S., Jones, D. \& Degens, H., Hysteresis in muscle

Rand, R. H., see Pender

Reartes, W., see Chialva

Rech, P. C., see Wiggers

Redjepov, S., see Uguz

Ren, G. D., Zhou, P., Ma, J., Cai, N., Alsaedi, A. \& Ahmad, B., Dynamical response of electrical activities in digital neuron circuit driven by autapse

Ren, H.-P., Bai, C., Huang, Z.-Z. \& Grebogi, C., Secure communication based on hyperchaotic Chen system with time-delay

Ren, W., see $M a$

Renson, L., Barton, D. A. W. \& Neild, S. A., Experimental tracking of limit-point bifurcations and backbone curves using controlbased continuation

Rezk, A. A., see Sayed

Rizzi, A., see De Santis

Rocşoreanu, C. \& Sterpu, M., Approximations of the homoclinic orbits near a double-zero bifurcation with symmetry of order two

Romanic, K., see Alombah

Romanovski, V. G., see Xiong

Ros, J., see Amador

Rosalie, M., see Changaival

Roulet, J. \& Mindlin, G. B., A diagrammatic representation of phase portraits and bifurcation diagrams of two-dimensional dynamical systems

Ruivo, E. L. P. \& de Oliveira, P. P. B., Inferring the limit behavior of some elementary cellular automata

Ruks, L. \& Van Gorder, R. A., On the inverse problem of competitive modes and the search for chaotic dynamics
1750181

1750192

1750040

1750135

1750001

1750087

1730004

1730015

1730011

1750132

1730003

1730016

1730044

1730023

1750083

1750187

1750076

1750098

1730002

1730004

1750211

1750109

1750067

1750126

1730022

1750215

1730045

1730028

1730032
Ryashko, L., see Bashkirtseva

1750208

1730014

1750208

1750211

1750037

1730015

Sah, M. PD., see Rajamani

Sahoo, B., Panda, L. N. \& Pohit, G., Stability, bifurcation and chaos of a traveling viscoelastic beam tuned to 3:1 internal resonance and subjected to parametric excitation

Saifuddin, M., Samanta, S., Biswas, S. \& Chattopadhyay, J., An eco-epidemiological model with different competition coefficients and strong-Allee in the prey

Samanta, S., see Ghosh

Samanta, S., see Saifuddin

Sanjuán, M. A. F., see López

Sayed, W. S., Fahmy, H. A. H., Rezk, A. A. \& Radwan, A. G., Generalized smooth transition map between tent and logistic maps

Seo, J. M., see Moon

Seo, J., see Kim

Seoane, J. M., see López

Severino, R., see Falcão

Shao, X., see Lei

Sharma, S. \& Kumari, N., Backward bifurcation in a Cholera model: A case study of outbreak in Zimbabwe and Haiti

She, Z., see Cheng

She, Z., see Jiang

Shen, Q. \& Liu, W., A novel digital image encryption algorithm based on orbit variation of phase diagram

Shen, Y.-Q., see Glimm

Shoreh, A. A.-H., see Mahmoud

Shu, S., see $X u$

Sieber, J., see Thompson

Siegmund, S., see Garay

Silva, L., Leonel Rocha, J. \& Silva, M. T., Bifurcations of 2-periodic nonautonomous stunted tent systems

Silva, M. T., see Silva

Simpson, D. J. W. \& Tuffley, C. P., Subsumed homoclinic connections and infinitely many coexisting attractors in piecewiselinear maps

Simpson, D. J. W., Grazing-sliding bifurcations creating infinitely many attractors

Sirakoulis, G. Ch., see Dourvas

Smale, S., see Rajapakse

Soares, M. J., see Falcão

Söderbacka, G., see Osipov

1750017

1730027

1750167

1730027

1750223

1730004

1750176

1750006

1750223

1730039

1750052

1750170

1750026

1750222

1750204

1750003

1750144

1750062

1730048

1750057

1730020

1730020

1730010

1730042

1750089

1750132

1730039

1750116

1750033

1730013

Sornette, D., see Yukalov

Sprott, J. C. \& Hoover, W. G., Harmonic oscillators with nonlinear damping

1730037

Sprott, J. C., see $L i$

1750149

Sprott, J. C., see $L i$

1750160

1750023 
Sprott, J. C., see Nazarimehr

1750037

Sprott, J. C., see Wei

Sprott, J. C., see Xiong

Stankevich, N. V., Kuznetsov, N. V., Leonov, G. A. \& Chua, L. O., Scenario of the birth of hidden attractors in the Chua circuit

Starkov, K. E. \& Jimenez Beristain, L., Dynamic analysis of the Melanoma model: From cancer persistence to its eradication

Sterpu, M., see Rocşoreanu

Stewart, I., Spontaneous symmetry-breaking in a network model for quadruped locomotion

$\mathrm{Su}, \mathrm{Y}$., see Liao

Sui, S. \& Li, B., Bounding the number of zeros of Abelian integral for a class of integrable non-Hamilton system

Sulalitha Priyankara, K. G. D., Balasuriya, S. \& Bollt, E., Quantifying the role of folding in nonautonomous flows: The unsteady double-gyre

Sun, C., Chen, Z. \& Xu, Q., Generating a double-scroll attractor by connecting a pair of mutual mirror-image attractors via planar switching control

Sun, H.-R., see Yang

Sun, J., Liao, X., Chen, X. \& Guo, S., Privacyaware image encryption based on logistic map and data hiding

Sun, K., see Liu

Sun, L., see Guo

Sun, X. J., see Yan

Sun, X., Li, Y. \& Xiao, Y., A predatorprey model with prey population guided antipredator behavior

Sun, X., Yu, P. \& Qin, B., Global existence and uniqueness of periodic waves in a population model with density-dependent migrations and Allee effect

Sun, Z., see Zhang

Susin, A., see Ferrer

Suzuki, H., see Hirakawa

Taha, A.-K., see Leonel Rocha

Tahir, F. R., Abdul-Hassan, K. M., Abdullah, M. A., Pham, V.-T., Hoang, T. M. \& Wang, $\mathrm{X}$., Analysis and stabilization of chaos in permanent magnet DC motor driver

Tajaddodianfar, F., see Luo

Tanino, T., see Tatsumi

Tatsumi, K. \& Tanino, T., A perturbation based chaotic system exploiting the quasiNewton method for global optimization

Tatsumi, K., A weaker sufficient condition for the chaoticity of extended perturbation-based updating system for global optimization

Tavakoli, H., see Hajipour

Teka, W. W., see Upadhyay

Thamilmaran, K., see Leo Kingston

Thompson, J. M. T., Hutchinson, J. W. \& Sieber, J., Probing shells against buckling: A
1730008

1750128

1730038

1750151

1750109

1730049

1750013

1750196

1750197

1750015

1750073

1750171

1750124

1750112

1750099

1750192

1750148

1750005

1730040

1730030

1750173

1750203

1750047

1750047

1750085

1750198

1730019

1730025 nondestructive technique for laboratory testing

1730048

Thompson, J. M. T., Instabilities of elastic and spinning systems: Concepts and phenomena

1730029

Tian, C. J., Chaos in the sense of Devaney for two-dimensional time-varying generalized symbolic dynamical systems

Tian, C. J., Hypercyclicity for a class of discrete spatiotemporal systems

Tian, R. L., Zhao, Z. J., Yang, X. W. \& Zhou, Y. F., Subharmonic bifurcation for a nonsmooth oscillator

Tigan, G., Llibre, J. \& Ciurdariu, L., Degenerate fold-Hopf bifurcations in a Rössler-type system

Tiwari, P. K., see Ghosh

Tomimura, Y., see Matsushita

Trostorff, S., see Garay

Tuffley, C. P., see Simpson

Uguz, S., Redjepov, S., Acar, E. \& Akin, H., Structure and reversibility of $2 D$ von $\mathrm{Neu}-$ mann cellular automata over triangular lattice

Upadhyay, R. K., Mondal, A. \& Teka, W. W., Mixed mode oscillations and synchronous activity in noise induced modified MorrisLecar neural system

Valenzuela, L. M., Falconi, M. \& Blé, G., A generalist predator and the planar zero-Hopf bifurcation

Van Gorder, R. A., see Adusumilli

Van Gorder, R. A., see Ruks

van Veen, L., see Buono

Vieira, J. C. C., see Marcondes

Virot, E., Faranda, D., Amandolese, X. \& Hémon, P., Chaotic dynamics of flags from recurring values of flapping moment

Volos, C., see Pham

Volos, C., see Pham

Volos, C., see Pham

Wan, X., see Zhang

Wan, X., see Zhang

Wang, A., see Xu

Wang, C. H., Xia, H. \& Zhou, L., A memristive hyperchaotic multiscroll jerk system with controllable scroll numbers

Wang, C., see Lin

Wang, C., see Zhou

Wang, D., see Liang

Wang, D., see Li

Wang, D., see Li

Wang, H. \& Li, X., Infinitely many heteroclinic orbits of a complex Lorenz system

Wang, J., Lu, B., Liu, S. \& Jiang, X., Bursting types and bifurcation analysis in the preBötzinger complex respiratory rhythm neuron

Wang, J., see Li
1750060

1750045

1750163

1750068

1750167

1750101

1750057

1730010

1750083

1730019

1750034

1730007

1730032

1750136

1750175

1750020

1750053

1750097

1750138

1750096

1750140

1730046

1750091

1750159

1750027

1750071

1750224

1750131

1750110

1750010 1750018 
Wang, J., see $W u$

Wang, J., see Zhou

Wang, L. \& Yang, X.-S., The coexistence of invariant tori and topological horseshoe in a generalized Nosé-Hoover oscillator

Wang, L., Li, N., Lei, F. \& Chu, Z., Topological entropy and mixing invariant extremal distributional chaos

Wang, L., Zhao, Y., Gao, Y. \& Liu, H., Chaos to multiple mappings

Wang, L., see Jiang

Wang, L., see Wang

Wang, L., see Xu

Wang, M., see Lin

Wang, W., Qiao, Y., Miao, J. \& Duan, L., Dynamic analysis of fractional-order recurrent neural network with Caputo derivative

Wang, X., Akgul, A., Cicek, S., Pham, V.-T. \& Hoang, D. V., A chaotic system with two stable equilibrium points: Dynamics, circuit realization and communication application

Wang, X., see Pham

Wang, X., see Tahir

Wang, X., see Wang

Wang, X., see $W u$

Wang, X., see Xiong

Wang, X., see Zhang

Wang, Y., Ma, J., Xu, Y., Wu, F. \& Zhou, P., The electrical activity of neurons subject to electromagnetic induction and Gaussian white noise

Wang, Y., see Xu

Wang, Y., see Yuan

Wang, Y.-X., see $M a$

Wang, Z., Wang, X., Li, Y. \& Huang, X., Stability and Hopf bifurcation of fractionalorder complex-valued single neuron model with time delay

Wang, Z., Xu, Z., Mliki, E., Akgul, A., Pham, V.-T. \& Jafari, S., A new chaotic attractor around a pre-located ring

Wang, Z., Zhang, J., Wang, L. \& Liu, Y., Bifurcation analysis of the oblique shock wave in the supersonic flow over a wedge from a calculus of variation approach

Wang, Z., see Cang

Wang, Z., see Wei

Waurick, M., see Garay

Wei, J., see Wei

Wei, X. \& Wei, J., Stability and bifurcation analysis in the photosensitive CDIMA system with delayed feedback control

Wei, Z. C., Moroz, I., Sprott, J. C., Wang, Z. \& Zhang, W., Detecting hidden chaotic regions and complex dynamics in the selfexciting homopolar disc dynamo

Wei, Z., see Nazarimehr

Wen, Y., see Qiu
1750165

1750021

1750111

1750139

1750119

1750108

1750048

1730046

1750159

1750181

1750130

1750097

1750173

1750209

1750127

1750128

1750074

1750030

1730046

1750028

1750011

1750209

1750152

1750048

1750024

1730008

1750057

1750177

1750177

1730008

1750221

1750040
Wen, Z., Bifurcations and exact traveling wave solutions of the celebrated Green-Naghdi equations

Wesson, E., see Pender

1750114

1730016

Wiggers, V. \& Rech, P. C., Chaos, periodicity, and quasiperiodicity in a radio-physical oscillator

Wiggins, S., see Demian

Wiggins, S., see Lopesino

Wu, A., see Cang

Wu, C., see Yang

Wu, F., see Wang

Wu, P., Li, C., He, X. \& Huang, T., A memristor-based Lorenz circuit and its stabilization via variable-time impulsive control

Wu, R., see Liu

Wu, X., Ding, X., Lu, T. \& Wang, J., Topological dynamics of Zadeh's extension on upper semi-continuous fuzzy sets

Wu, X., Wang, X. \& Chen, G., On the large deviations theorem of weaker types

$\mathrm{Wu}, \mathrm{X} .$, A remark on topological sequence entropy

Xia, H., see Wang

Xia, L., see Dong

Xiang, S., see Zhang

Xiao, P., see $D u$

Xiao, Y., see Sun

Xie, D., see Jin

Xie, J., see Chen

Xiong, A., Sprott, J. C., Lyu, J. \& Wang, X., $3 D$ printing - The basins of tristability in the Lorenz system

Xiong, T., see $L v$

Xiong, Y., Han, M. \& Romanovski, V. G., The maximal number of limit cycles in perturbations of piecewise linear Hamiltonian systems with two saddles

Xu, C., Li, C., Lü, J. \& Shu, S., On the network analysis of the state space of discrete dynamical systems

$\mathrm{Xu}, \mathrm{C}$., see Yang

Xu, H., Chen, F. \& Jin, W., Topological conjugacy classification of elementary cellular automata with majority memory

$\mathrm{Xu}$, H., Li, Y., Zhang, J., Han, H., Zhang, B., Wang, L., Wang, Y. \& Wang, A., Ultrawideband chaos life-detection radar with sinusoidal wave modulation

Xu, M., Katori, Y. \& Aihara, K., Stability analysis on sparsely encoded associative memory with short-term synaptic dynamics

$\mathrm{Xu}, \mathrm{Q}$., see Sun

$\mathrm{Xu}, \mathrm{X}$., see Zhou

$\mathrm{Xu}, \mathrm{Y}$., see $L i$

1750031

1730026

1750165

1750127

1750107

1750091

1750214

1750169

1750122

1750099

1750120

1750078

1750128

1750121

1750126

1750062

1750199

1750217

1730046

$\mathrm{Xu}, \mathrm{Y}$., see Wang

$\mathrm{Xu}, \mathrm{Y}$., see Yang

$\mathrm{Xu}, \mathrm{Z}$., see Wang
1730034

1750197

1750193

1750160

1750030

1750150

1750152 
Xue, Y., see Guo

Yadav, C. K., see Gupta

Yajima, T., see Yamasaki

Yalcin, M. E., see Goncu

Yamamoto, Y., see Hirakawa

Yamasaki, K. \& Yajima, T., KCC analysis of the normal form of typical bifurcations in one-dimensional dynamical systems: Geometrical invariants of saddle-node, transcritical, and pitchfork bifurcations

Yan, D. \& Fu, X., Long-time behavior of spatially and size-structured population dynamics with delayed birth process

Yan, F., see $L i$

Yan, H. \& Sun, X. J., Impact of partial time delay on temporal dynamics of WattsStrogatz small-world neuronal networks

Yan, J., Kang, X. \& Yang, L., The trade-off mechanism in mammalian circadian clock model with two time delays

Yan, S. \& Guo, S., Bifurcation phenomena in a Lotka-Volterra model with cross-diffusion and delay effect

Yang, A., see Zhang

Yang, B.-X., Sun, H.-R. \& Feng, Z., Unilateral global bifurcation, half-linear eigenvalues and constant sign solutions for a fractional Laplace problem

Yang, H., see Duan

Yang, H., see $L i$

Yang, L., see Yan

Yang, N., Xu, C., Wu, C., Jia, R. \& Liu, C., Modeling and analysis of a fractional-order generalized memristor-based chaotic system and circuit implementation

Yang, S., see Jin

Yang, W., see $M a$

Yang, X. W., see Tian

Yang, X.-D., see $Y u$

Yang, X.-S., see Wang

Yang, Y., Zhang, T., Xu, Y. \& Zhou, J., A delayed virus infection model with cell-to-cell transmission and CTL immune response

Yang, Y.-X., see Gao

Yang, Z., see Jiang

Yi, C., see Zhang

Yi, C., see Zhang

Yi, W., see $L v$

Yin, Z., Chaotic dynamics of composition operators on the space of continuous functions

Yu, D., see Zhou

$\mathrm{Yu}, \mathrm{M}$., see Liu

$\mathrm{Yu}, \mathrm{P}$., see Jiang

Yu, P., see Sun

$\mathrm{Yu}, \mathrm{S}$., see $\mathrm{He}$

$\mathrm{Yu}, \mathrm{S}$., see Lin

Yu, S., see Qiu
1730047

1750056

1750145

1730017

1730040

1750145

1750032

1750194

1750112

1750147

1750105

1750155

1750015

1750025

1750016

1750147

1750199

1750120

1750158

1750163

1750035

1750111

1750150

1750123

1750125

1750086

1750096

1750121

1750084

1750193

1750171

1750043

1750192

1750146

1750106

1750040
Yu, T.-J., Zhang, W. \& Yang, X.-D., Nonlinear dynamics of flexible L-shaped beam based on exact modes truncation

1750035

1750051

1750162

Yuan, L., see Li

Yuan, R., Jiang, W. \& Wang, Y., Nonresonant double Hopf bifurcation in toxic Phytoplankton-Zooplankton model with delay

1750028

Yuan, Z., Li, H., Miao, Y., Hu, W. \& Zhu, X., Digital-analog hybrid scheme and its application to chaotic random number generators

1750210

Yukalov, V. I., Yukalova, E. P. \& Sornette, D., Dynamic transition in symbiotic evolution induced by growth rate variation

Yukalova, E. P., see Yukalov

Zeng, C., see Lin

Zhang, B., see Lü

Zhang, B., see Xu

Zhang, C., see Han

Zhang, F., Liao, X., Chen, Y.-A., Mu, C. \& Zhang, G., On the dynamics of the chaotic general Lorenz system

Zhang, F., Wang, X., Liao, X., Zhang, G. \& $\mathrm{Mu}, \mathrm{C} .$, Dynamical behaviors of a modified Lorenz-Stenflo chaotic system

Zhang, G., see Zhang

Zhang, G., see Zhang

Zhang, H., Ding, H. \& Yi, C., Design-oriented analysis of slow-scale bifurcations in single phase $D C-A C$ inverters via autonomous transformation approach

Zhang, H., Li, W., Ding, H., Luo, P., Wan, X. \& Hu, W., Nonlinear modal analysis of transient behavior in cascade $D C-D C$ boost converters

Zhang, H., Luo, P. \& Ding, H., Bifurcation analysis of charged particles moving on a rough surface under different damping effects

Zhang, H., Wan, X., Li, W., Ding, H. \& Yi, C., Observer-pattern modeling and slowscale bifurcation analysis of two-stage boost inverters

Zhang, J., see Glimm

Zhang, J., see Wang

Zhang, J., see Xu

Zhang, K., see Zhang

Zhang, L. Y., Zhang, Y., Liu, Y., Yang, A. \& Chen, G., Security analysis of some diffusion mechanisms used in chaotic ciphers

Zhang, L., Pan, W., Mu, P., Li, X., Xiang, S., Zhang, W., Jiang, N. \& Li, N., The role of master laser with feedback in time-delay signature suppression of semiconductor laser subject to chaotic optical injection

Zhang, N., see Jin

Zhang, Q., see Zhou
1750169

1750120

1730013

1730013

1750207

1750172

1730046

1750051

1750075

1750074

1750074

1750075

1750086

1750140

1750069

1750096

1750003

1750048

1730046

1750212

1750155

1750021 
Zhang, S.-S., see Gao

Zhang, T. \& Li, J., Exact torus knot periodic orbits and homoclinic orbits in a class of three-dimensional flows generated by a planar cubic system

Zhang, T., see Liu

Zhang, T., see Li

Zhang, T., see Li

Zhang, T., see Yang

Zhang, W., Han, B., Zhang, K. \& Ding, Q., Dynamic analysis of a rotor system supported on squeeze film damper with air entrainment

Zhang, W., see Guo

Zhang, W., see $L i$

Zhang, W., see Wei

Zhang, W., see $Y u$

Zhang, W., see Zhang

Zhang, X., Constructing a chaotic system with any number of attractors

Zhang, X., Dynamics of nonautonomous ordinary differential equations with quasiperiodic coefficients

Zhang, Y., Dou, G., Sun, Z., Guo, M. \& Li, Y., Establishment of physical and mathematical models for $\mathrm{Sr}_{0.95} \mathrm{Ba}_{0.05} \mathrm{TiO}_{3}$ memristor

Zhang, Y., see Guo

Zhang, Y., see Guo

Zhang, Y., see Zhang

Zhao, C., see Lü

Zhao, X.-W., see Lai

Zhao, Y., see Luo
1750059

Zhao, Y., see Wang

1750119

Zhao, Z. J., see Tian

1750163

Zheng, F., see Lei

Zheng, X., see Jiang

1750205

1750133

1750194

1750080

1750150

1750212

1750124

1750224

1730008

1750035

1750169

1750118

1750092

1750148

1730047

1750124

1750155

1750172

1750142

1750179

Zhou, J., Xu, X., Yu, D. \& Li, W., Dynamics and control of a delayed oscillator network by pinning strategy

Zhou, J., see Yang

Zhou, L., Chen, Z., Wang, J. \& Zhang, Q., Local bifurcation analysis and global dynamics estimation of a novel 4-dimensional hyperchaotic system

1750052

1750222

1750193

1750150

Zhou, L., Wang, C. \& Zhou, L., Generating four-wing hyperchaotic attractor and two-wing, three-wing, and four-wing chaotic attractors in $4 D$ memristive system

Zhou, L., see Wang

Zhou, L., see Zhou

Zhou, P., see Ren

Zhou, P., see Wang

Zhou, Y. F., see Tian

Zhou, Y. \& Li, J., Exact solutions and dynamics of the Raman soliton model in nanoscale optical waveguides, with metamaterials, having polynomial law nonlinearity

1750188

Zhou, Y., see Liao

1750013

1750210

1750182

Zhusubaliyev, Z. T., see Churilov

Zotos, E. E., Basins of convergence of equilibrium points in the generalized Hill problem

Zou, R. \& Guo, S., Bifurcation of reaction cross-diffusion systems
1730043

1750049 